\title{
Scalar Properties of Transversely Isotropic Tuff from Images of Orthogonal Cross Sections
}

\author{
P. A. Berge \\ J. G. Berryman* \\ S. C. Blair \\ C. Peña \\ Lawrence Livermore National Laboratory, Livermore, California 94551-9900 \\ * Author for correspondence
}

This paper was prepared for submittal to the

This manuscript was prepared for submittal to the

Second International Conference on Imaging Technolgies:

Techniques and Civil Engineering Applications

Davos, Switzerland

May 25 - 30, 1997

January 1997

This is a preprint of a paper intended for publication in a journal or proceedings. Since changes may be made before publication, this preprint is made available with the understanding that it will not be cited or reproduced without the permission of the author. 


\section{DISCLAIMEa}

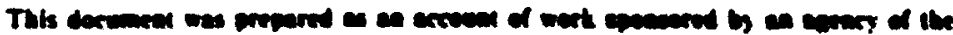

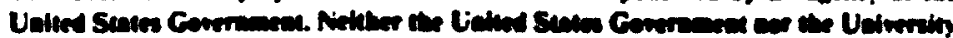

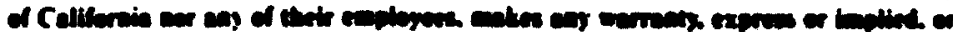

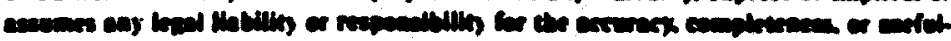

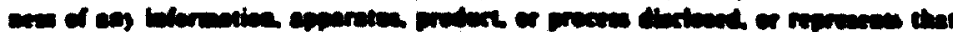

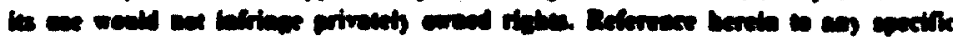

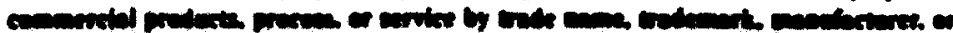

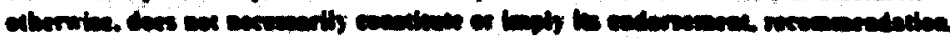

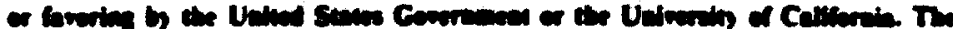

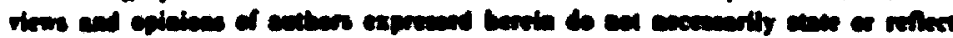

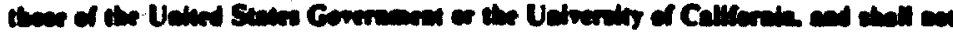

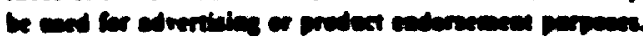




\title{
lersion date: January 6. 1997
}

Scalar Properties of Transversely Isotropic Tuff from Images of Orthogonal Cross Sections

\author{
P. A. Berge, J. G. Berryman," S. C. Blair, and C. Peña \\ Lawrence Livermore National Laboratory \\ P. O. Box 808 L-202 \\ Livermore, CA 94551-9900
}

-Author for correspondence. 


\title{
Scalar Properties of Transversely Isotropic Tuff from Images of Orthogonal Cross Sections
}

\author{
P. A. Berge, ${ }^{1}$ J. G. Berryman, ${ }^{1}$ S. C. Blair, ${ }^{1}$ and C. Peña ${ }^{1}$
}

\begin{abstract}
Image processing methods have been used very effectively to estimate physical properties of isotropic porous earth materials such as sandstones. Anisotropic materials can also be analyzed in order to estimate their physical properties, but additional care and a larger number of well-chosen images of cross sections are required to obtain correct results. Although low-symmetry anisotropic media present difficulties for two-dimensional image processing methods, geologic materials are often transversely isotropic. Scalar properties of porous materials such as porosity and specific surface area can be determined with only minor changes in the analysis when the medium is transversely isotropic rather than isotropic. For example, in a rock that is transversely isotropic due to thin layers or beds, the overall porosity may be obtained by analyzing images of cross sections taken orthogonal to the bedding planes, whereas cross sections lying within the bedding planes will determine only the local porosity of the bed itself. It is known for translationally invariant anisotropic media that the overall specific surface area can be obtained from radial averages of the two-point correlation function in the full three-dimensional volume. Layered materials are not translationally invariant in the direction of the layering, but we show nevertheless how averages of cross sections may be used to obtain the specific surface area for a transversely isotropic rock. We report values of specific surface area obtained for thin sections of Topopah Spring Tuff from Yucca Mountain, Nevada. This formation is being evaluated as a potential host rock for geologic disposal of nuclear waste. Although the present work has made use of thin sections of tuff for the images, the same methods of analysis could also be used to simplify quantitative analysis of three-dimensional volumes of pore structure data obtained by means of $\mathrm{x}$-ray microtomography or other methods, using only a few representative cross sections chosen from the full three-dimensional data set. This approach provides a quick and easy way to get initial estimates of physical properties that can later be refined using more time-consuming and computationally intensive methods.
\end{abstract}

\footnotetext{
${ }^{1}$ Lawrence Livermore National Laboratory, P. O. Box 808, Livermore, California 94551-9900
} 


\section{Introduction}

Image processing methods provide a relatively inexpensive means of measuring and/or estimating physical properties of rocks. Two-dimensional methods as applied to optical photographs and other, nonoptical types of 2.0 scans of cross sections are well-known and continue to provide important information that may not be available from any other source (Blair et al.. 1996). Three-dimensional methods based on x-ray tomographic images of rocks are just coming online (Coker et al. 1996: Roberts et al.. 1996). Another recent three-dimensional imaging technique uses high-resolution laser scanning and confocal microscopy (Fredrich and Lindquist, 1996). Most of the analysis to date on image processing methods for rock analysis has concentrated on isotropic rocks, but it is clear that many rocks of interest are highly anisotropic. So one of the next phases of research on image processing techniques wi: necessarily be to address the issue of anisotropy and determine to what extent reliable information about physical properties can be obtained from such methods. The present paper is a first step in that direction.

We summarize results of characterization of microstructure for three samples of welded tuff material. Cross sections of the samples were examined and analyzed using digitized image processing techniques. Spatial correlation functions were used to determine the specific surface areas and porosities of the SEM (Scanning Electron Microscope) images, assuming the tuff material is a two-phase medium consisting of pores and cracks in a welded matrix.

Three samples of welded tuff from Yucca Mountain, Nevada, were analyzed. Samples were from depths of 1122.6, 1146.5, and 1153 to 1153.6 feet. As in previous work by Blair et al. (1996), horizontal and vertical thin sections were produced for analyzing three samples of tuff material. Sample 17348, taken from 1153-1153.6 feet of depth, is represented by 5 SEM images at 49, 500, 700 , and $1000 \times$ magnifications in the parallel direction with respect to the core axis and 2 SEM images at 500x magnification in the perpendicular direction. Sample 17350 , taken at 1146.5 feet, is represented by 9 SEM images at 200 , $500,700 \times$ magnifications in the parallel direction and 6 SEM images at 500 and $700 \times$ magnifications in the perpendicular direction. Sample 17351, taken at 1122.6 feet, is represented by 4 SEM images at $700 \times$ magnification in the parallel direction and 2 SEM images at 690 and $700 \times$ magnifications in the perpendicular direction.

\section{Methods}

The tuff samples are treated as two-phase materials consisting of pores and cracks embedded in a welded matrix, where one- and two-point correlation functions can be applied to study the material. A simple indicator function $f$ can be used to assign either 1 or 0 to any spatial position $x$ in the digital image of the material. The function $f(x)=1$ if $x$ is in the pore or crack space and $f(x)=0$ if $x$ is in the solid material. The binary image itself represents the indicator func- 
tion $f(x)$, while the mean of the function $f$ (i.e.. the sum of all $f$ values in the image divided by the total number of pixels) is the one-point correlation function $\because_{1}$. The value of $s_{1}$ obtained from the image cross-sectional area provides an estimate of the porosity 0 .

The two-point correlation function $S_{2}$ is defined as the porbability that two points separated by a known distance will both lie in the pore space of the medium (Blair et al.. 1996). The two-point correlation function is computed by analyzing a digitized image of the tuff material, comparing pairs of pixels at different separation distances and incrementing a sum when both pixels are in the pore space. The one- and two-point correlation functions are defined as:

$$
S_{1}=\langle f(x)\rangle=\phi
$$

and

$$
S_{2}(r)=\langle f(x) f(x+r)\rangle .
$$

The angular brackets $<\cdot>$ denote the volume (or, in the case of plane images, the areal) average over all positions $x$. For a more complete discussion of the two-point correlation function and the algorithm, see Torquato (1980), Berryman (1985), Berryman and Blair (1986), Blair and Berryman (1992), and Blair et al. (1996).

The mean pore area was measured by image processing and analysis software (Ultimage). A scanned image is converted into a binary image, where pixels in the pore space were assigned a value of 1 and pixels in the solid matrix a value of 0 . The number of pixels in the pore space is counted and summed; this value is equal to the pore area, since one pixel is equal to one square unit. All the detectable pore areas in a binary image are determined and the mean pore area is calculated from the detectable pores in the image at the resolution available.

Debye et al. (1957) and Berryman and Blair (1986) have shown that for isotropic porous materials the slope of the two-point correlation function near the origin (zero lag) is proportional to the specific surface area of that porous material:

$$
S_{2}^{\prime}(0)=-\frac{s}{4}
$$

where the specific surface area $s$ is the total surface area of the pore-grain interface per total volume of the porous material. The specific surface area follows from this equation for any statistically isotropic porous material regardless of the particle shapes. Furthermore, Berryman (1987) has shown that even for anisotropic materials a similar formula applies if the left hand side of the equation is interpreted as the radial average of the anisotropic two-point correlation function. To provide some qualitative understanding of the result, consider the behavior of the correlation product $f(x) f(x+r)$ near the interface for very small values of $\operatorname{lag} r$. If $x$ is in the pore space but just outside a particle, a small change 
in $r$ directed towards the particle boundary will result in a change of this product from 1 rol). Taking the derivative t urns these steps into delta functions and then integrating them over the entire volume produces a value proportional to the inface area. We recommend reading the references to gain more quantitative insight into the meaning of (3).

The correlation function approach is not limited in application to spherical particle assemblages. However. it is sometimes convenient to consider penetrable and impenetrable sphere assemblages since various exact results or numerical results are available for such materials. It is known (Torquato, 1980) that the penetrable sphere model results in a smooth and monotically decreasing $S_{2}$ that varies from $O$ at zero lag to $\phi^{2}$ at a lag equal to the twice the radius of the monodisperse penetrable spheres. The impenetrable sphere model results in an oscillatory $S_{2}$ that varies from $\phi$ at zero lag to $\phi^{2}$ at infinite lag, with a first minimum at approximately twice the radius of the impenetrable spheres. It will prove convenient to compare results obtained on real rocks to these idealized cases if only for the sake of providing a means of distinguishing the variety of material behaviors observed in nature.

\section{Results for Sample Porosity}

As described in previous work by Blair et al. (1996), horizontal and vertical thin sections were produced for analyzing three samples of tuff material. Image processing was applied to obtain the two-point correlation function to determine the porosity, specific surface area, and the mean pore area of a given cross-sectional area of welded tuff material in a binary image format. The rock core sample appeared anisotropic and cross-sectional images of the core, both perpendicular and parallel to the core axis, were taken to determine the heterogeneous state of the material. Several images from each sample, parallel and perpendicular, were recorded. Images of magnification $500 \times$ or greater were used to tabulate the results to be discussed.

The welded tuff material appeared to be anisotropic with many pores, cracks, and fractures in various shapes and sizes. Although all the images have different pore formations, most images have large isolated pores that consist of either pores or cracks and a multitude of microscopic pores that dominate the microstructure of the image.

Images from sample 17348 parallel to the core axis are from a tuff core taken at 1153 feet from Yucca Mountain. For this orientation, various magnifications of the same image were considered. We found in these images several isolated pores where the first has large pores that appear to be cracks with a multitude of smaller pores. Estimates of porosity range between 7 and $8 \%$. Images from sample 17348 perpendicular to the core axis were taken from the same core. These images differed with the presence of large cracks, resulting in the porosity ranging from 14 to $30 \%$.

Images from sample 17351 both perpendicular and parallel to the core axis are 


\section{Topopah Spring tuff}

\author{
(700x, perpendicular)
}

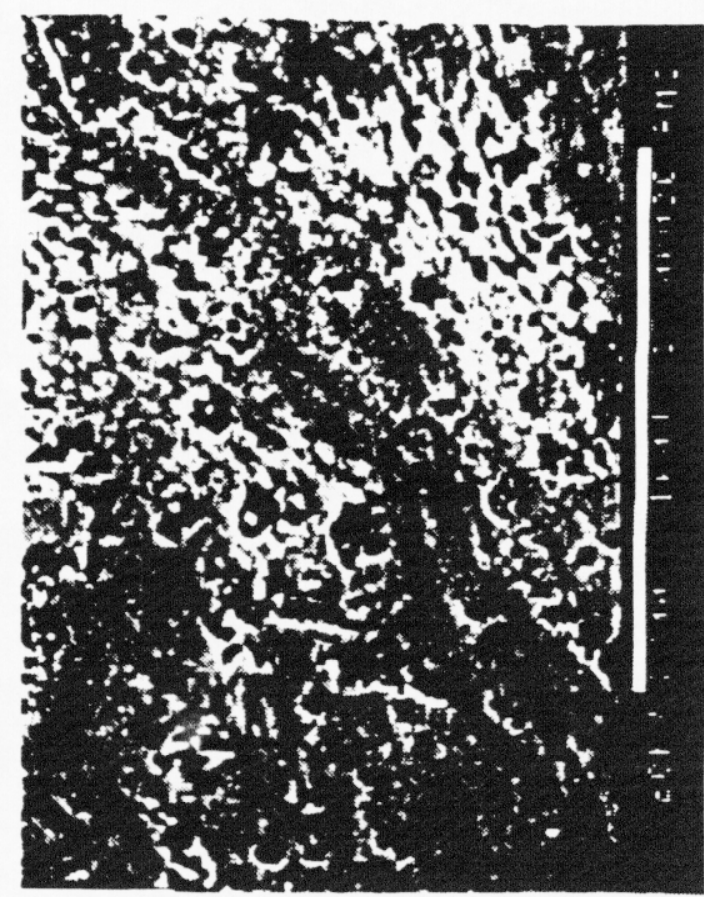

USW G4 1122.6' 07/13/93

- 0017351 perpendicular to core axi-

\section{Topopah Spring tuff}

\author{
$(700 \times$, parallel)
}

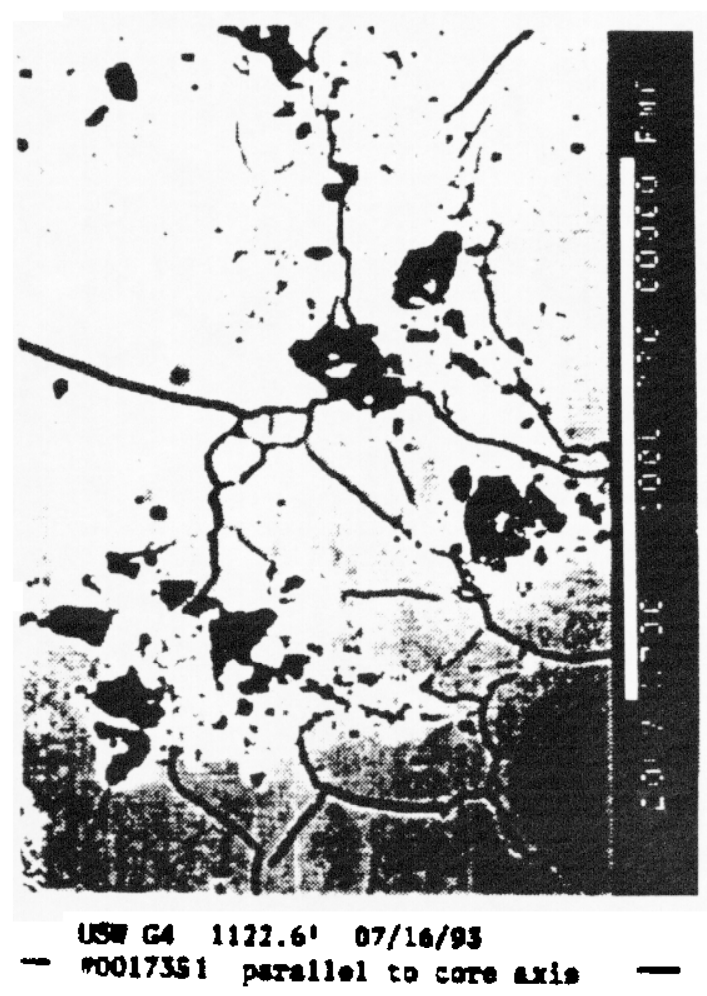

Figure 1: Perpendicular and parallel cross sections of tuff sample 17351 from Yucca . Mountain, Nevada, at magnification $700 \times$.

from a tuff core taken from 1122.6 feet of depth at Yucca Mountain, examples of which are shown in Figure 1. The parallel cross section has various isolated pores, cracks, and fractures that run the length of the image. The porosity estimated for this sample orientation ranged from 11 to $20 \%$. For the perpendicular sample orientation, we find scattered isolated pores. Both samples considered have the same general appearance with porosity ranging from 13 to $15 \%$.

\section{Analysis of Correlation Functions and Other Measures}

In studying the two tuff samples considered here, our primary interest is determining the porosity, mean pore area, and specific surface area. From previous work, image processing was found to be a effective tool when studying the relationship between the physical properties and the pore structures of rocks. Not surprisingly, we found a wide variation in the microstructure of the tuff material that caused difficulty in describing the rock. Results of the analysis are displayed in Table 1.

The porosities were found from the two-point correlation function values for lag of zero. Table 1 shows that porosity ranges from 7 to $30 \%$ for these two sam- 
ples. The large range in porosity shows the hetrogeneous nature of the material, where images varied greatly in pore structure. For ample 17348. the porosities are lighty higher in the perpendicular direction. but for sample 17353 there was no noriceable difference between the perpendicular and parallel directions.

T.IBLE 1. Porosity. Specific Surface Area, and Mean Pore Area for Tuff Samples from Yucca llountain. levada.

\begin{tabular}{|r|r|r|c|c|c|}
\hline \hline ID\# & $\perp$ & Mag. & $\begin{array}{c}\text { Porosity (0) } \\
\text { Average } \pm \text { SD }\end{array}$ & $\begin{array}{c}\text { Specific Surface } \\
\text { Area }\left(\mu \mathrm{m}^{-1}\right) \pm \text { SD }\end{array}$ & $\begin{array}{c}\text { Mean Pore } \\
\text { Area }\left(\mu \mathrm{m}^{2}\right) \pm \mathrm{SD}\end{array}$ \\
\hline 17348 & $\|$ & 500 & $0.08 \pm 0.005$ & $0.0835 \pm 0.0076$ & $8.02 \pm 0.65$ \\
17348 & $\|$ & 700 & $0.07 \pm 0.050$ & $0.0962 \pm 0.0630$ & $4.44 \pm 2.39$ \\
17348 & $\|$ & 1000 & $0.12 \pm 0.042$ & $0.1844 \pm 0.0235$ & $3.91 \pm 1.82$ \\
\hline 17348 & $\perp$ & 500 & $0.30 \pm 0.014$ & $0.1753 \pm 0.0059$ & $31.81 \pm 1.38$ \\
17348 & $\perp$ & 500 & $0.14 \pm 0.007$ & $0.1442 \pm 0.0065$ & $11.61 \pm 0.85$ \\
\hline 17351 & $\|$ & 700 & $0.14 \pm 0.035$ & $0.1658 \pm 0.0356$ & $10.91 \pm 2.18$ \\
17351 & $\|$ & 700 & $0.19 \pm 0.024$ & $0.2273 \pm 0.0188$ & $7.67 \pm 0.43$ \\
17351 & $\|$ & 700 & $0.11 \pm 0.007$ & $0.1224 \pm 0.0091$ & $12.95 \pm 2.33$ \\
17351 & $\|$ & 700 & $0.20 \pm 0.027$ & $0.2469 \pm 0.0202$ & $8.44 \pm 0.65$ \\
\hline 17351 & $\perp$ & 690 & $0.13 \pm 0.006$ & $0.1682 \pm 0.0125$ & $8.12 \pm 0.11$ \\
17351 & $\perp$ & 700 & $0.15 \pm 0.008$ & $0.1946 \pm 0.0029$ & $7.96 \pm 0.17$ \\
\hline \hline
\end{tabular}

The averaged mean pore areas for each set of images are also shown in Table 1. The range of mean pore areas for both samples is from 3.91 to $31.81 \mu \mathrm{m}^{2}$ when taking into account all of the pore space area of the image scanned. There are no noticeable differences between the mean pore area for images in the perpendicular and parallel directions: apparently neither direction governs mean pore area. As in previous discussions, many images contain large isolated pores. As a result, the pore-size distributions for many images are bimodal. The distributions including just the smaller pore sizes are of interest due to their tendency to control micropores. In order to exclude the large pore size distribution, the threshold from the image processing software was reduced until the particle numbers, which is itself a function of the pore area, was $90 \%$ of the original particle count.

The $S_{2}$ function can give an estimate of effective pore size, mean grain size, and specific surface area of a sample being analyzed. Here, we are interested in the specific surface area of the tuff material. The specific surface values given in Table 1 were computed using $S_{2}$. As described earlier, the slope of two-point 
correlation function at zero lag is proportional to the specific surface area of the iample: $\therefore, 20)=-\therefore$ t.

Table l displays the results for the tuff sample 1734.3. Raw images parallel to the core axis have a few large isolated pores in contrast to the smaller undistinsubhed pures. The $\dot{S}_{2}$ function for this case (not shown) suggests that the image mimics a penetrable sphere model. The $S_{2}$ curve approaches $0^{2}$ quickly, showing that the pore space above $1.5 \mu \mathrm{m}$ is uncorrelated. The specific surface area of this image is $0.08 \mu \mathrm{m}^{-1}$. Table 1 shows that higher magnification images yield higher values of the specific surface area. This is due to the higher resolution being able to detect the rougher surfaces. The mean pore area size for this image was found to be approximately $8 \mu \mathrm{m}^{2}$. The pore size distribution follows a bimodal distribution with $90 \%$ of the pores smaller than $12 \mu \mathrm{m}^{2}$. This distribution is important because later we will see that even though the mean pore area for a given image may be large. $90 \%$ of the particles will tend to be less than $1.5 \mu \mathrm{m}^{2}$. indicating their tendency to control the microstructure of the material.

Sample 17343 perpendicular to the core axis was studied using a couple of images with large differences in porosity and mean pore area. We found that most pores tend to be less than $15 \mu \mathrm{m}^{2}$, again supporting the hypothesis that the smaller pores control the microstructure of the material. The $S_{2}$ curve in Figure 2 mimics the penetrable sphere model and the other shown in Figure 3 mimics the impenetrable sphere model. Therefore, the example in Figure 2 shows that the pore space is correlated up to $75 \mu \mathrm{m}$, indicating the various large pores, whereas in Figure 3 the $S_{2}$ curve approaches $\phi^{2}$ quickly and has an uncorrelated pore space above $10 \mu \mathrm{m}$.

Sample 17351 parallel to the core axis is represented by four images with a porosity range of 11 to $20 \%$. Here we found images that differed in structure, which then affects the correlation functions. The $S_{2}$ curve in Figure 4 indicates that the pore space is correlated up to $50 \mu \mathrm{m}$. Even though the $S_{2}$ curves were similar, the difference is pronounced in the porosity and specific surface area values.

Sample 17351 perpendicular to the core axis is represented by two images that are similar in porosity, specific surface area, and mean pore area. The $S_{2}$ curve in Figure 5 again mimics the penetrable sphere model, where the pore space is uncorrelated above $10 \mu \mathrm{m}$ and it approaches $\phi^{2}$ very quickly.

\section{Discussion}

As mentioned earlier, the two-point correlation function can give an estimate of porosity, effective pore size, mean grain size, and specific surface area. In our analysis, we were primarily concerned with the specific surface area of the material in order to improve our understanding of the pore-grain interface and its effect on the permeability. According to previous work by Blair and Berryman (1987) on sandstones, the specific suraface area of a given material could accurately be measured using the slope method, since $S_{2}^{\prime}(0)=-s / 4$. For the two 


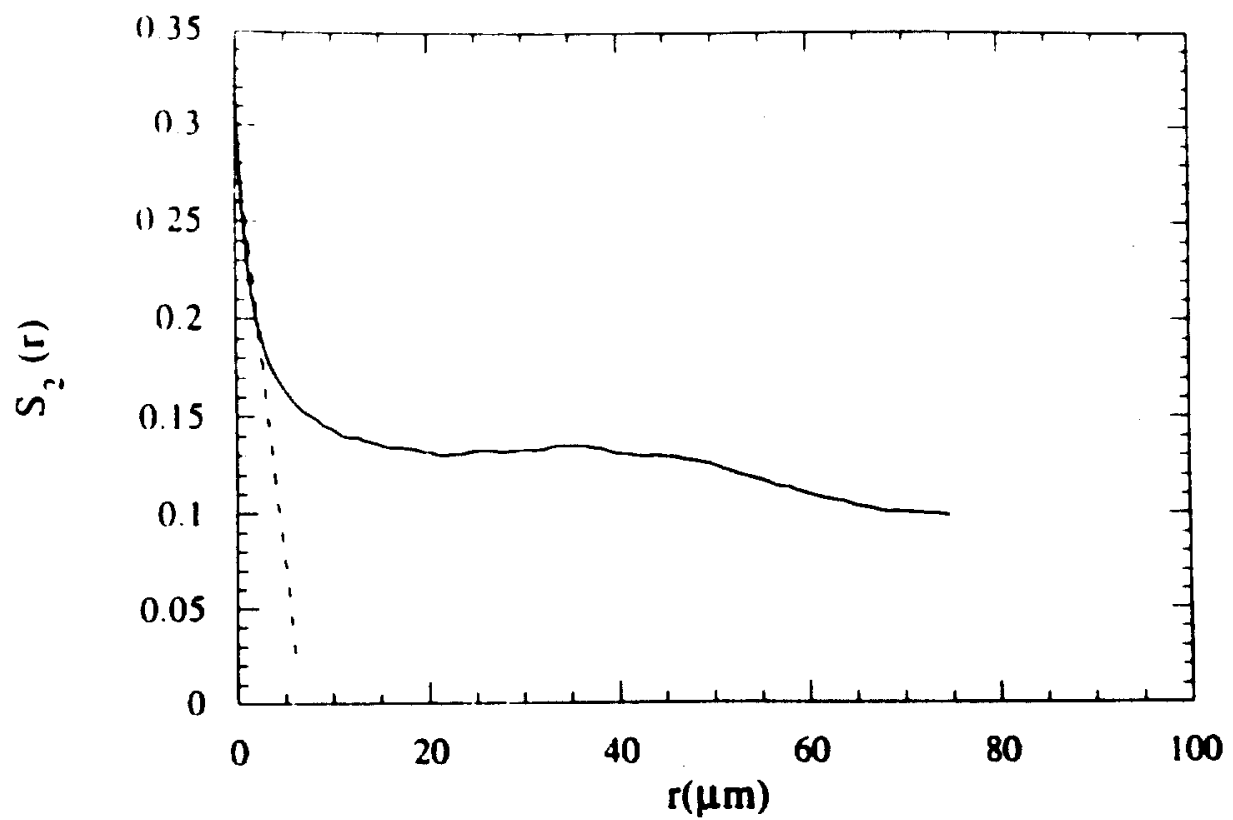

Figure 2: Correlation function (solid curve) for welded tuff sample 17348 at perpendicular (in plane) orientation. Slope near the origin is indicated by one dahsed line. The other dashed line shows the asymptotic value $\phi^{2}$.

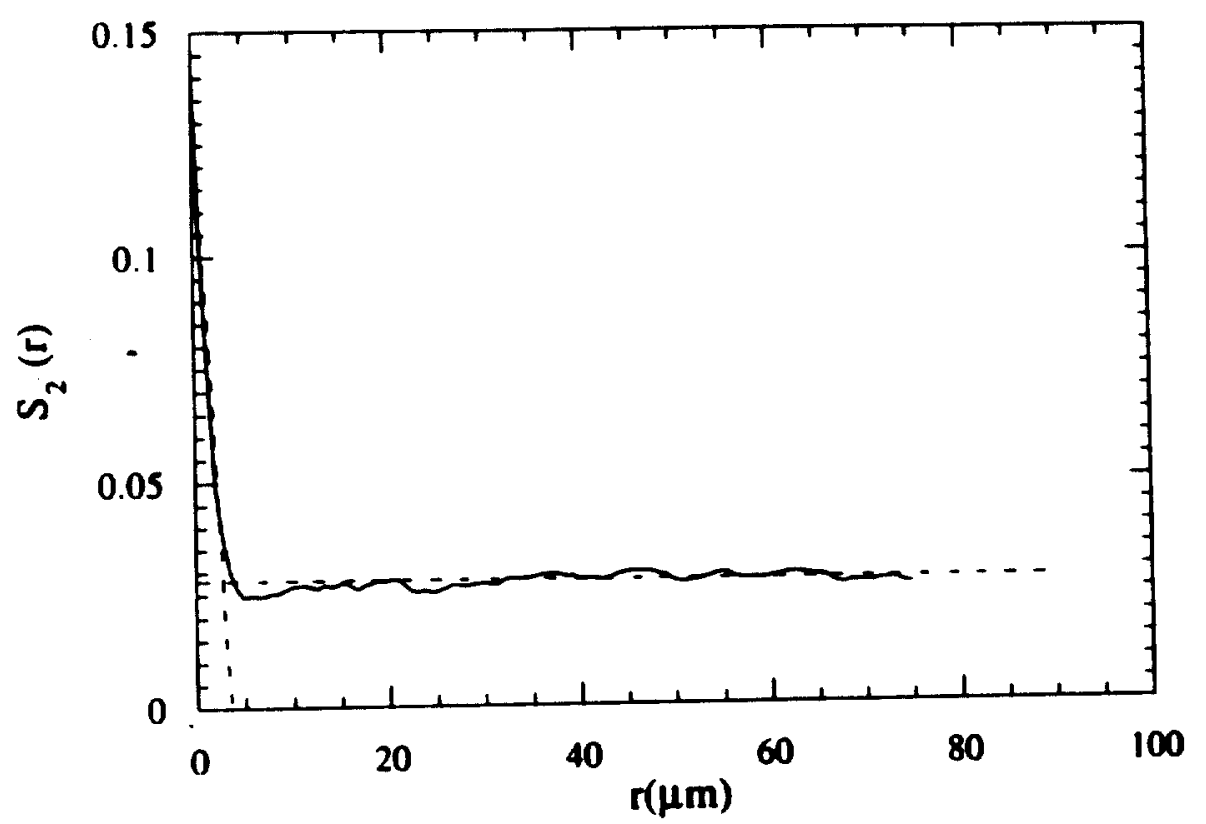

Figure 3: Same as Figure 2 for a different part of the same sample. 


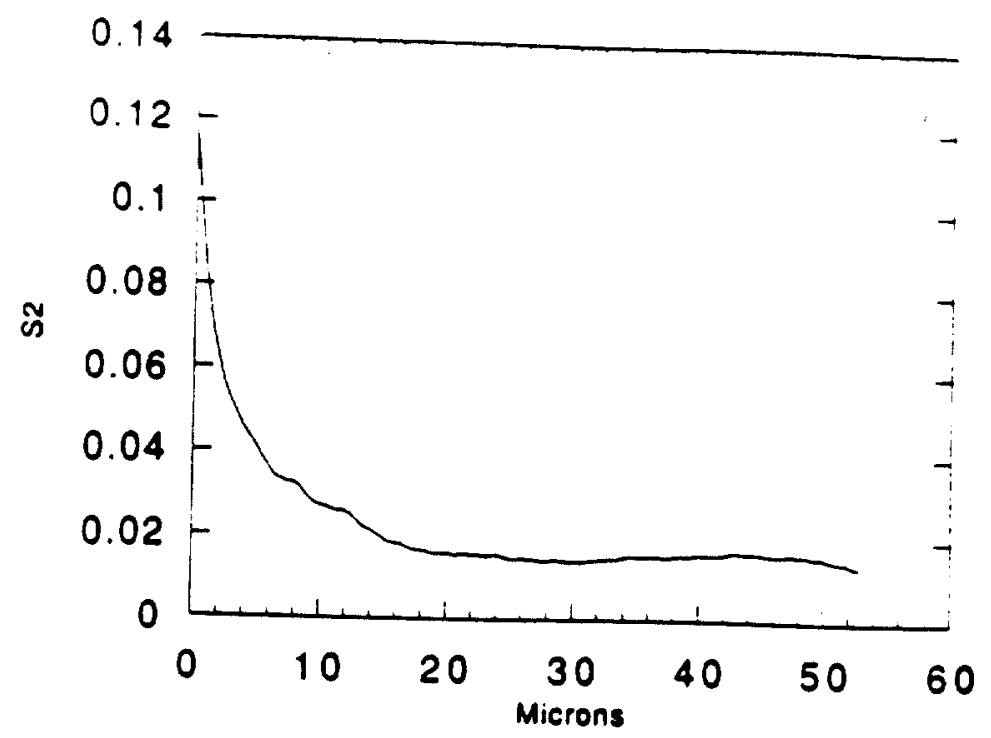

Figure 4: Correlation function (solid curve) for welded tuff sample 17351 in parallel (along core axis) orientation.

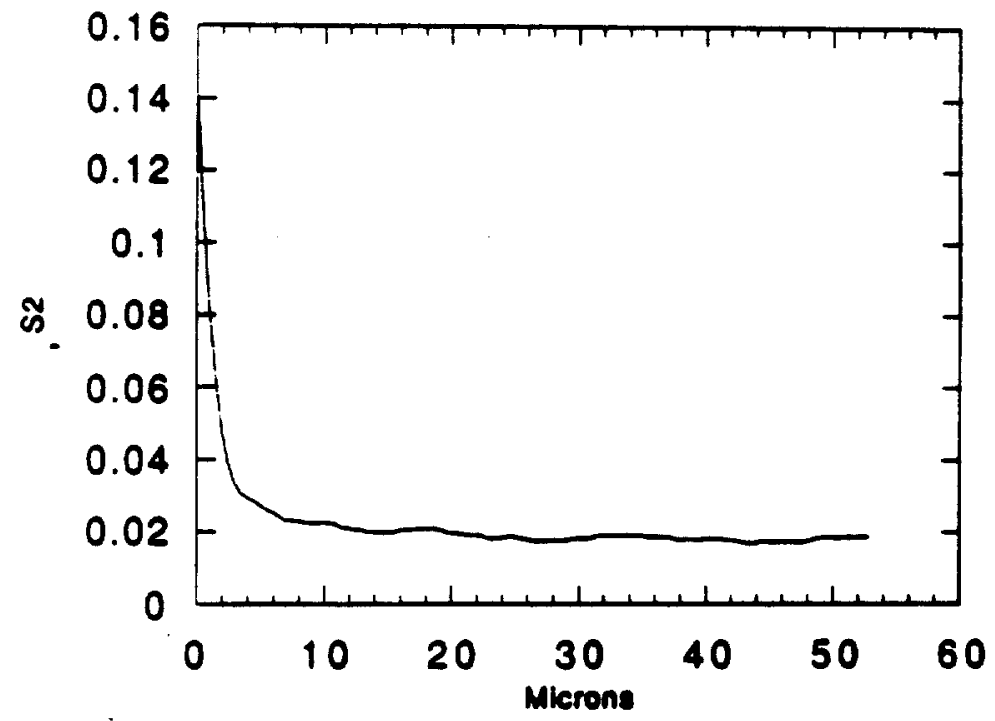

Figure 5: Same as Figure 4 for perpendicular (in plane) orientation. 


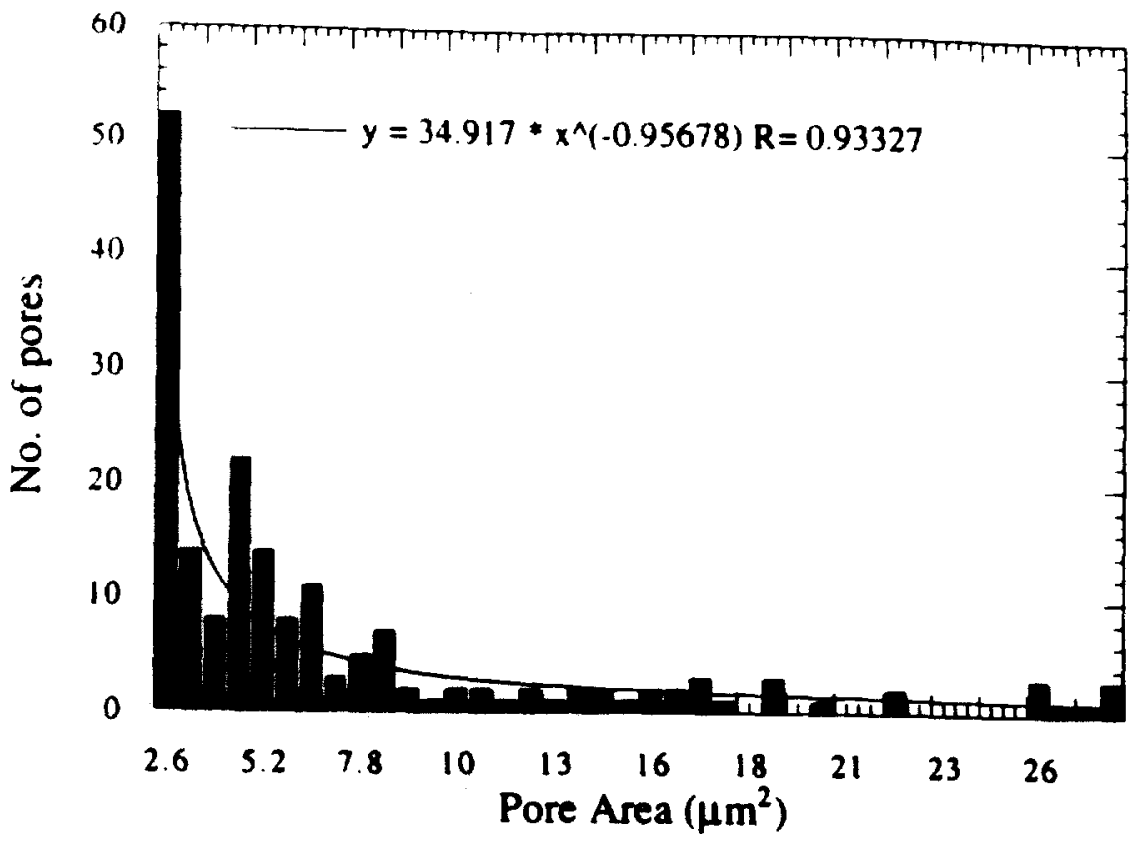

Figure 6: Pore area histogram for matrix material of welded tuff sample 17351, using smallest $90 \%$ of the detectable pore distribution.

samples in the horizontal and vertical directions, we found a large variability in specific surface area values. This range of values was found to be from 0.0835 to $0.2469 \mu \mathrm{m}^{-1}$. This large range (which has been found to be as much as an order of magnitude for some materials) measures the variability within the rock itself and was independent of both magnification and orientation of the samples.

The results generated from the tuff material analysis are more easily understood when contrasted against previous work generated from synthetic and natural sandstones. According to Blair et al. (1996), natural sandstones are expected to have higher specific surface area values than synthetic ones since the grains in the natural sandstones tend to be much rougher in appearance than those of glass beads. Furthermore, in keeping with this line of thinking, we can easily conclude that the specific surface area values for the tuff material will be much higher and have a larger range of variability than will the natural sandstones, because the material is inhomogeneous with cracks, fractures, and pores of varied sizes and shapes.

In the synthetic sandstones, Blair et al. (1996) show that the specific surface area ranges from 0.0162 to $0.0253 \mu \mathrm{m}^{-1}$. The natural sandstones have a larger range due to the rougher grains of 0.0756 to $0.1330 \mu \mathrm{m}^{-1}$, while the tuff samples have the greatest range and values of 0.0382 to $0.2978 \mu \mathrm{m}^{-1}$. The higher specific surface area values for the tuff images in the perpendicular direction indicate that there is more structure in the perpendicular direction, since at higher resolution the roughness is more easily resolved. Typically, as the magnification increases 
the measured pperific surface area value increases as well. Again. we emphasize the extent to which the tutf rock is highly anisot copic.

Perhaps the most interesting new feature of the present analysis is the mean pure area. In our analysis. the mean pore area was estimated using image proc...ing " "ware in which all detectable pores are measured from the binary image and lhe mean pore area is calculated from this set of detectable pores. As Table I indicates. the values for the mean pore area varied greatly with sample and orientation. Overall the mean pore area ranged from a low of $3.9 \mu \mathrm{m}^{2}$ to a high of $3.3 .18 \mu \mathrm{m}^{2}$ when taking into account all of the pore space area of the image scanned. As mentioned earlier. the larger isolated pores that many images contain result in these images having bimodal pore-size distributions. and the distributions with just the smaller pore sizes are of independent interest due to their tendency to control the microstructure of the material. Results show that the few larger pores often dominate the total distribution. To obtain more detail of the matrix distribution of smaller pores, we analyzed $90 \%$ of the original detectable particles in an image, which was found to be a function of the pore area.

We found that by taking $90 \%$ of the original particle count in many images the mean pore areas were less than $10 \mu \mathrm{m}^{2}$ and most detectable pores were less than $20 \mu \mathrm{m}^{2}$. This result indicated that the microstructure of the matrix is indeed controlled by the smaller micropores in the image. The information provided by the smaller pores is important for hydrological and geochemical processes not covered in the present work. The full detectable pore area indicates a mean pore area of $11.2 \mu \mathrm{m}^{2}$ and a bimodal distribution. Figure 6 shows the result obtained with a distribution of $90 \%$ of the original particle count. We find that the distribution for the smaller pores estimate a lower mean pore area for the image of $5.95 \mu \mathrm{m}^{2}$ and the pore distribution follows a power law distribution as shown in Figure 6. Similar results were observed for the other samples studied.

\section{Conclusions}

From this study we conclude that, using image processing techniques, we can determine quantitative measures of microstructure for the two analyzed samples of welded tuff. Pore surface areas were generally less than $10 \mu \mathrm{m}^{2}$ and followed power law distributions. The specific surface area has a wide range of values and is greater than that observed for some nominally clean sandstones. The tuff material is neither homogeneous nor isotropic, as is clearly seen in Figure 1.

\section{Acknowledgments}

Work performed under the auspices of the U. S. Department of Energy by the Lawrence Livermore National Laboratory under contract No. W-7405-ENG48 and supported in part by the Geosciences Research Program of the DOE Office of Energy Research within the Office of Basic Energy Sciences, Division of Engineering and Geosciences. 
References

Berryman. J. (i. 1 198.) ). ".easurement of spatial correlation functions using image proressing techniques." J. Appl. Phys.. 57. 2:374-2384.

Berryman. J. (i, 1957). "Relationship between specific surface area and spatial currolation functions for anisotropic porous media," J. Wath. Phys.. 28. 24t24.5.

Berryman. J. G., and Blair. S. C. (1986). "Lse of digital image analysis to estimate fluid permeability of porous materials: Application of two-point correlation functions," J. Appl. Phys., 60, 1930-1938.

Berryman. J. G., and Blair, S. C. (1987). "Kozeny-Carman relations and imageprocessing methods for estimating Darcy's constant," J. Appl. Phys., 62, $2221-2228$.

Blair. S. C., and Berryman, J. G. (1992). "Permeability and relative permeability in rocks," in Fault Mechanics and Transport Properties in Rocks, Proceedings of the Brace Symposium. MIT, June 10-11, 1990, Cambridge, MA, B. Evans and T.-f. Wong (eds.), Academic Press, London, Chapter 7. pp. 169-186.

Blair, S. C., Berge, P. A., and Berryman, J. G. (1996). "Using two-point correlation functions to characterize microgeometry and estimate permeabilities of synthetic and natural sandstones," J. Geophys. Res., 101, 20359-20375.

Coker, D. A., Torquato, S., and Dunsmuir, J. H. (1996). "Morphology and physical properties of Fontainebleu sandstone via a tomographic analysis," $J$. Geophys. Res., 101, 17497-17506.

Debye, P., Anderson, H. R., Jr., and Brumberger, H. (1957). "Scattering by an inhomogeneous solid. II. The correlation function and its application," $J$. Appl. Phys., 28, 679-683.

Fredrich, J..T., and Lindquist, W. B. (1996). "Statistical characterization of the three-dimensional microgeometry of porous media and correlation with macroscopic transport properties," Eos, Transactions of the American Geophysical Union, 77, Fall Meeting Supplement, p. F735.

Roberts; J. J., Bonner, B. P., Duba, A. G., and Schneberk, D. L. (1996). "Physical properties of preserved core from The Geysers scientific corehole," SB-15D: Proceedings of the 21st Annual Stanford Workshop on Geothermal Reservoir Engineering, Stanford University, pp. 313-317.

Torquato, S. (1980). Microscopic Approach to Transport in Two-Phase Random Media, PhD thesis, State University of New York at Stony Brook, Department of Mechanical Engineering, Stony Brook, New York, December, 1980. 


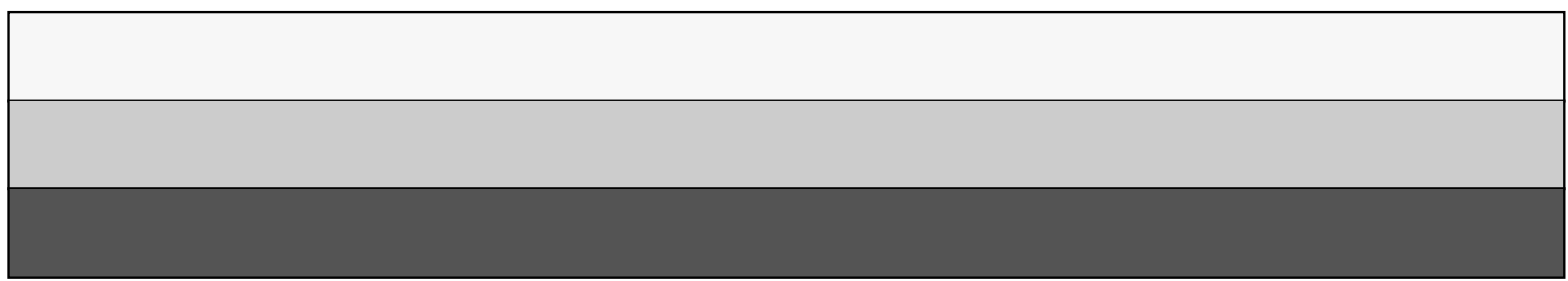

